\title{
Editorial: Compound Climate Extremes in the Present and Future Climates: Machine Learning, Statistical Methods and Dynamical Modelling
}

\begin{abstract}
Wei Zhang ${ }^{1 *}$, Hiroyuki Murakami ${ }^{2,3}$, Abdou Khouakhi ${ }^{4}$ and Ming Luo ${ }^{5,6}$
${ }^{1}$ Department of Plants, Soils and Climate, Utah State University, Logan, UT, United States, ${ }^{2}$ University Corporation for Atmospheric Research (UCAR), Boulder, CO, United States, ${ }^{3}$ Geophysical Fluid Dynamics Laboratory, National Oceanic and Atmospheric Administration, Princeton, NJ, United States, ${ }^{4}$ School of Water, Energy and Environment, Centre for Environmental and Agricultural Informatics, Cranfield University, Cranfield, United Kingdom, ${ }^{5}$ School of Geography and Planning, Sun Yat-sen University, Guangzhou, China, ${ }^{6}$ Guangdong Provincial Engineering Research Center for Public Security and Disaster, and Guangdong Key Laboratory for Urbanization and Geo-Simulation, Guangzhou, China
\end{abstract}

Keywords: compound extremes, machine learning, statistical methods, dynamical modeling, climate change

\section{OPEN ACCESS}

Edited and reviewed by: Yuqing Wang, University of Hawaii at Manoa, United States

${ }^{*}$ Correspondence: Wei Zhang w.zhang@usu.edu

Specialty section: This article was submitted to Atmospheric Science, a section of the journal

Frontiers in Earth Science

Received: 01 November 2021 Accepted: 04 November 2021 Published: 19 November 2021

Citation: Zhang W, Murakami H, Khouakhi A and Luo M (2021) Editorial: Compound Climate Extremes in the Present and Future Climates: Machine Learning,

Statistical Methods and

Dynamical Modelling.

Front. Earth Sci. 9:807224. doi: 10.3389/feart.2021.807224

\section{Editorial on the Research Topic}

Compound Climate Extremes in the Present and Future Climates: Machine Learning, Statistical Methods and Dynamical Modelling

Compound extremes, namely simultaneous, concurrent, or coincident extreme hydrometeorological events, may have broader impacts on human society and the environment than any individual extreme alone (Hao et al., 2018; Zscheischler et al., 2018, 2020, 2021; AghaKouchak et al., 2020; Raymond et al., 2020). There are a wide range of compound events that occur on a variety of spatial and temporal scales: typical examples include droughts coupled with heat waves, coastal flooding coupled with wind hazards, sea level rise and storm surge, or tropical cyclones followed by heat waves. However, we are yet to fully understand all types of compound extremes, the dynamical and physical processes associated with their occurrence, the framework and the methods required for their analysis, and their likelihood within the present and future climate.

This research topic aims to advance our knowledge about the processes and dynamical linkages associated with different types of compound extremes, to showcase the development of new statistical methods and machine learning techniques for efficiently examining these extremes, and to quantify the potential risks of compound extremes in the present and future climate. This Research Topic comprises 15 articles (14 research articles and 1 review article) on extreme weather events and compound extremes. These articles have visited compound hydrometeorological events, extreme temperatures, extreme weather and climate events in tropical ocean basins, climate modeling and machine learning technologies for examining extremes and their compounding effects.

Compound hydrometeorological events under present and future climates have been examined in this research topic. Giang et al. used pattern scaling along with the Generalized Extreme Value (GEV) distribution to calculate changes in multi-day extreme precipitation in North Central Vietnam in future projections under three Representative Concentration Pathways from IPCC AR5. Similarly, an attribution analysis over the mid-lower reaches of the Yangtze River of China found that anthropogenic warming has reduced the likelihood of 2019-like 14-days heavy precipitation by $20 \%$, but increased that of 2 -days extremes by $30 \%$ (Nanding et al.). Wu et al. found that the fraction of 
extreme precipitation events in Guangdong preceded by hot weather is even larger in more populated and urbanized areas such as the Pearl River Delta (PRD) region, and revealed significant increases in the frequency and fraction of compound extreme heat and precipitation events. Lai et al. examined the evolution of compound flood days based on global precipitation and storm surge data in observations and reported that the frequency has risen significantly on the east coast of the US and northern Europe, but decreased significantly in southern Europe and Japan. Zhang et al. reviewed the physical drivers, mechanisms and methods related to compound hydrometeorological extremes and discussed current advances in the understanding of compound heat wave and drought (hot-dry), compound heat stress and extreme precipitation (hot-wet), and coldwet, cold-dry and compound flooding. Yang et al. investigated the responses of heat stress to temperature and humidity changes due to anthropogenic heating and urban expansion in South and North China. Using gauge-based precipitation observations, they found that heavy precipitation events were the main contributor to the increasing trend of summer precipitation over the Three-Rivers Headwater Region (Zhao et al.). Li et al. unraveled synoptic weather patterns that modulate warm-sector heavy rainfall in South China. They found that the locations of six large-scale extreme precipitation events were related to the urban agglomerations in Guangdong-Hong Kong-Macao Greater Bay Area.

Two papers in this Research Topic have advanced the understanding of extreme temperatures. Ngarukiyimana et al. examined the spatial and temporal variations of daily maximum and minimum surface air temperature (Tmin and Tmax) and diurnal temperature range. With high confidence, the results indicate a significant positive trend in both Tmin and Tmax in three study regions in Rwanda during the whole study period. Based on observations of automatic weather stations in Beijing during the summers of 2014-2020, Zong et al. studied the interaction between heat waves events and the Urban Heat Island (UHI) effect and found that latent heat flux has increased more in rural areas because of sufficient water availability and vegetation.

Studies in this Research Topic have also focused on developing machine learning technologies for examining extremes. For example, Huang et al. reported that the stacking model and the XGBoost model performed the best in predicting solar radiation. Wang et al. developed a new extreme detection method for unravelling remote compound extremes in Southeast China. Wang et al. introduced the MultiLLR ML model and found that the subseasonal prediction skill of China precipitation with $2-6$ weeks lead time could be

\section{REFERENCES}

AghaKouchak, A., Chiang, F., Huning, L. S., Love, C. A., Mallakpour, I., Mazdiyasni, O., et al. (2020). Climate Extremes and Compound Hazards in a Warming World. Annu. Rev. Earth Planet. Sci. 48, 519-548. doi:10.1146/ annurev-earth-071719-055228

Hao, Z., Singh, V., and Hao, F. (2018). Compound Extremes in Hydroclimatology: A Review. Water 10, 718. doi:10.3390/w10060718 enhanced. Finally, the T-mode principal component analysis statistical approach was used to study the warm-sector heavy rainfall by unravelling large-scale circulation patterns ( $\mathrm{Li}$ et al.).

Works in this Research Topic have also examined extreme weather and climate events in tropical ocean basins. For example, footprints of the Atlantic Multidecadal Oscillation (AMO) in the western North Pacific assessed using observations and coupled atmosphere-ocean simulations of sea surface temperature (WNP SST) and atmospheric states exert a significant impact on tropical cyclone intensity (Sun et al.). Chen et al. compared the temporal evolution of environmental conditions for Rapid Intensification (RI) and non-RI of TCs in the South China Sea (SCS) during 2000-2018, and identified key factors for RI of SCS TCs using the box difference index and stepwise regression.

Several future research directions for compound climate extremes are documented: 1) projecting the risk of compound climate extremes corresponding to future warming; 2 ) evaluating the impacts of the compound climate extremes on natural and built environments; 3) designing adaptation measures to the changing risk of the extremes; 4) improving subseasonal-toseasonal prediction of these compound extremes; 5) improving the representation and evaluation of compound climate extremes in fully-coupled climate models; and 6) applying machine learning and other advanced methods to understand these extremes.

\section{AUTHOR CONTRIBUTIONS}

All authors listed have made a substantial, direct, and intellectual contribution to the work and approved it for publication.

\section{FUNDING}

WZ is supported by USDA NIFA Hatch Project (1026229), the UAES Seed Grant and the startup fund of Utah State University. ML is supported by the Pearl River Talent Recruitment Program of Guangdong Province, China (2017GC010634) and the Science and Technology Program of Guangzhou, China (202102020489).

\section{ACKNOWLEDGMENTS}

We appreciate the Editorial Board and the Editorial Office of Frontiers in Earth Science for their kind invitation to edit this Research Topic and their support throughout the entire process.

Raymond, C., Horton, R. M., Zscheischler, J., Martius, O., AghaKouchak, A., Balch, J., et al. (2020). Understanding and Managing Connected Extreme Events. Nat. Clim. Chang. 10, 611-621. doi:10.1038/s41558-020-0790-4

Zscheischler, J., Martius, O., Westra, S., Bevacqua, E., Raymond, C., Horton, R. M., et al. (2020). A Typology of Compound Weather and Climate Events. Nat. Rev. Earth Environ. 1, 333-347. doi:10.1038/s43017-020-0060-Z

Zscheischler, J., Sillmann, J., and Alexander, L. (2021). Introduction to the Special Issue: Compound Weather and Climate Events. Weather Clim. Extremes, 100381. doi:10.1016/j.wace.2021.100381 
Zscheischler, J., Westra, S., Van Den Hurk, B. J. J. M., Seneviratne, S. I., Ward, P. J., Pitman, A., et al. (2018). Future Climate Risk from Compound Events. Nat. Clim Change 8, 469-477. doi:10.1038/s41558-018-0156-3

Conflict of Interest: The authors declare that the research was conducted in the absence of any commercial or financial relationships that could be construed as a potential conflict of interest.

Publisher's Note: All claims expressed in this article are solely those of the authors and do not necessarily represent those of their affiliated organizations, or those of the publisher, the editors and the reviewers. Any product that may be evaluated in this article, or claim that may be made by its manufacturer, is not guaranteed or endorsed by the publisher.

Copyright $\odot 2021$ Zhang, Murakami, Khouakhi and Luo. This is an open-access article distributed under the terms of the Creative Commons Attribution License (CC BY). The use, distribution or reproduction in other forums is permitted, provided the original author(s) and the copyright owner(s) are credited and that the original publication in this journal is cited, in accordance with accepted academic practice. No use, distribution or reproduction is permitted which does not comply with these terms. 